\title{
Solution of a Problem of Rubel Concerning Iteration and Algebraic Differential Equations
}

\section{WALter BergWeILER}

ABSTRACT. It is shown that there does not exist a transcendental entire function with the property that all its iterates satisfy the same algebraic differential equation. This answers a question of L. A. Rubel.

1. Introduction and main results. An analytic function $f$ is called differentially algebraic if it satisfies an algebraic differential equation; that is, an equation of the form

$$
P\left(z, f(z), f^{\prime}(z), \ldots, f^{(n)}(z)\right)=0
$$

where $P$ is a polynomial in $n+2$ variables that does not vanish identically. A function is called hypertranscendental (or transcendentally transcendental) if it is not differentially algebraic. For a survey of results and problems concerning hypertranscendental and differentially algebraic functions, we refer to three papers of Rubel $[17,18,19]$.

A family of functions is called uniformly differentially algebraic (or coherent) if there exists an algebraic differential equation which is satisfied by all functions of the family. Rubel [19, Problem 27] asked whether there exists a transcendental entire function $f$ such that the family $\left\{f^{k}\right\}$ of iterates of $f$ is uniformly differentially algebraic. The purpose of this paper is to answer Rubel's question.

Theorem 1. There does not exist a transcendental entire function whose iterates are uniformly differentially algebraic.

We note that the corresponding result for polynomials is known: the iterates of a polynomial are uniformly differentially algebraic if and only if the polynomial 
is conjugate (by a linear function) to a monomial, a Chebychev polynomial, or the negative of a Chebychev polynomial; compare [19, p. 663], see also [3].

Suppose now that the entire function $f$ has a repelling fixed point; that is, there exists $\zeta$ such that $f(\zeta)=\zeta$ and $\left|f^{\prime}(\zeta)\right|>1$. Define $\lambda=f^{\prime}(\zeta)$. Then there exists an entire function $\varphi$ such that $\varphi(0)=\zeta$ and

$$
\varphi(\lambda z)=f(\varphi(z)) .
$$

It is unique if we normalize it by $\varphi^{\prime}(0)=1$. We call $\varphi$ the Poincaré function to $f$ at $\zeta$. It is also common to name these functions after Schröder or Kœenigs. (The equation (2) was considered first by Schröder [20], Kœnigs [14] proved that it has a solution $\varphi$ analytic in a neighborhood of 0 if $\lambda \neq 0$ and $|\lambda| \neq 1$, and Poincaré [15] observed that (2) permits analytic continuation of $\varphi$ to the whole plane if $|\lambda|>1$.) For a further discussion of this and related equations, as well as their role in complex dynamics, we refer to [2, Chapter 6], [7, Chapter II], and [22, Chapter 3].

Boshernitzan and Rubel [5, Theorem 6.1] proved that $\varphi$ is differentially algebraic if and only if $\left\{f^{k}\right\}$ is uniformly differentially algebraic. Thus the following result is an immediate consequence of Theorem 1.

Theorem 2. Poincaré functions to transcendental entire functions are hypertranscendental.

Baker [1] proved that the repelling fixed points of the iterates of $f$ are dense in the Julia set of $f$, which is a non-empty (and perfect) set. In particular, some iterate has a repelling fixed point. (In fact, any $f^{k}, k \geq 2$, has this property, but we do not need this result proved in [4] here.) Combining the fact that some iterate of $f$ has a repelling fixed point with the result of Boshernitzan and Rubel quoted above, we see that Theorem 2 also implies Theorem 1; that is, the two theorems are actually equivalent.

We remark that Poincaré functions may also be defined for rational functions, in which case they are meromorphic. (Here and in the following "meromorphic" always means "meromorphic in the plane".) Ritt [16] determined all differentially algebraic Poincaré functions to rational functions. They are given explicitly in terms of the exponential, the cosine, or the Weierstraß $\wp$-function.

As already mentioned, the equation (2) still has a solution $\varphi$ if $0<|\lambda|<1$, but then $\varphi$ is not entire but defined only in some neighborhood of $\zeta$. The results of Boshernitzan and Rubel include this case and thus we find that $\varphi$ is hypertranscendental in this case, too. A similar remark applies to the solutions of the Böttcher and Abel equations, which correspond to the cases $\lambda=0$ and $\lambda=1$ - see [3] for the rational case.

The proof of our results is very similar to Ritt's proof [16]. In fact, many of his ideas are just repeated here, but I hope that the reader will appreciate this rather than reading words like "analogous" and "similar" too often. 
Ritt's basic idea is to assume that $\varphi$ is differentially algebraic, say

$$
P\left(z, \varphi(z), \varphi^{\prime}(z), \ldots, \varphi^{(n)}(z)\right)=0,
$$

and to substitute (2) in (3). This yields a new algebraic differential equation for $\varphi$. If the rank (defined in Section 4 below) of (3) is minimal, then the new equation is a multiple of the old one, and this then sharply reduces the possible forms of (3). We proceed similarly and substitute (2) in (3). This yields a new differential equation for $\varphi$, but its coefficients are transcendental if $f$ is transcendental. A theorem of Steinmetz [21], however, permits us to replace this new differential equation by another one which does have rational coefficients. This makes Ritt's approach, with suitable modifications, work in the transcendental case, too.

2. Steinmetz's theorem. We assume that the reader is familiar with the basic definitions and results of Nevanlinna theory; see [12] or [13]. The theorem of Steinmetz [21, Satz 1] referred to in the introduction is the following.

Lemma 1. Let $F_{0}, F_{1}, \ldots, F_{m}$ be not identically vanishing meromorphic functions and let $h_{0}, h_{1}, \ldots, h_{m}$ be meromorphic functions that do not all vanish identically. Let $g$ be a nonconstant entire function and suppose that there exists a positive constant $K$ such that

$$
\sum_{j=0}^{m} T\left(r, h_{j}\right) \leq K T(r, g)+S(r, g),
$$

where $S(r, g)=o(T(r, g))$ as $r \rightarrow \infty$ outside some exceptional set of finite measure. Suppose also that

$$
F_{0}(g) h_{0}+F_{1}(g) h_{1}+\cdots+F_{m}(g) h_{m}=0 .
$$

Then there exist polynomials $P_{0}, P_{1}, \ldots, P_{m}$ that do not all vanish identically such that

$$
P_{0}(g) h_{0}+P_{1}(g) h_{1}+\cdots+P_{m}(g) h_{m}=0 .
$$

Generalizations and different proofs of this result have been given by Brownawell [6] and Gross and Osgood [9, 10, 11].

3. The order of Poincaré functions. We shall use the following result concerning the order of growth of Poincaré functions.

Lemma 2. Poincaré functions to transcendental entire function have infinite order. 
If we combine this lemma with the well-known result that entire solutions of first-order algebraic differential equations have finite order [24, p. 110], then we see that Poincaré functions of transcendental entire functions cannot satisfy first order algebraic differential equations.

We remark, however, that the method of Section 5 and Section 6 could also be used to determine the possible first order equations explicitly, as it was done by Ritt, and then it can be verified that none of them corresponds to a transcendental entire function $f$.

Proof of Lemma 2. Let $\varphi$ be a Poincaré function to the transcendental entire function $f$. Then $T(|\lambda| r, \varphi)=T(r, f(\varphi))$ by (2). On the other hand, we have

$$
\lim _{r \rightarrow \infty} \frac{T(r, f(\varphi))}{T(r, \varphi)}=\infty
$$

see $[8$, Theorem 1$]$ or $[12$, p. 54$]$. Hence

$$
\lim _{r \rightarrow \infty} \frac{T(|\lambda| r, \varphi)}{T(r, \varphi)}=\infty
$$

Thus, for any given $M>1$, we find $r_{0}$ such that $T(|\lambda| r, \varphi) \geq M T(r, \varphi)$ for $r \geq r_{0}$. It follows that

$$
T\left(|\lambda|^{k} r_{0}, \varphi\right) \geq M T\left(|\lambda|^{k-1} r_{0}, \varphi\right) \geq M^{2} T\left(|\lambda|^{k-2} r_{0}, \varphi\right) \geq \ldots \geq M^{k} T\left(r_{0}, \varphi\right)
$$

so that

$$
\log T\left(|\lambda|{ }^{k} r_{0}, \varphi\right) \geq k \log M-O(1)=\frac{\log M}{\log |\lambda|} \log \left(|\lambda|{ }^{k} r_{0}\right)-O(1) .
$$

This implies that the order of $\varphi$ is at least $\log M / \log |\lambda|$. The conclusion follows because $M$ can be chosen arbitrarily large.

Remark. Using similar arguments, Valiron [23, Section 48] proved that if $f$ is a polynomial of degree $d$, then $\varphi$ has order $\log d / \log |\lambda|$.

4. The rank of an equation. Let $f$ be meromorphic, $n \in \mathbb{N}=\{1,2,3, \ldots\}$, $r_{j} \in \mathbb{N}_{0}=\mathbb{N} \cup\{0\}$ for $j=1,2, \ldots, n$, and put $r=\left(r_{1}, r_{2}, \ldots, r_{n}\right)$. Suppose that $r_{n} \neq 0$ except possibly if $n=1$ and define $M_{r}[f]$ by

$$
M_{r}[f](z)=f^{\prime}(z)^{r_{1}} f^{\prime \prime}(z)^{r_{2}} \ldots f^{(n)}(z)^{r_{n}},
$$

with the convention that $M_{(0)}[f]=1$. We call $M_{r}[f]$ the differential monomial of rank $r$ in $f$. Furthermore, $n$ is called the order, $d(r)=r_{1}+r_{2}+\cdots+r_{n}$ the degree, and $w(r)=r_{1}+2 r_{2}+\cdots+n r_{n}$ the weight of $r$ (or of $M_{r}[f]$ ). 
Given $q=\left(q_{1}, q_{2}, \ldots, q_{m}\right)$ and $r=\left(r_{1}, r_{2}, \ldots, r_{n}\right)$ with $q_{m}, r_{n} \neq 0$, we say that $q<r$ if $m<n$, or if $m=n$ and there exists $k \in\{1,2, \ldots, n\}$ such that $q_{k}<r_{k}$ and $q_{j}=r_{j}$ for $k<j \leq n$. Of course, we write $q \leq r$ if $q<r$ or $q=r$.

We can write (1) in the form

$$
\sum_{q \leq r} a_{q}(z, f(z)) M_{q}[f](z)=0,
$$

where the $a_{q}$ are polynomials in two variables and where $a_{r}$ does not vanish identically. We may assume that $a_{r}=1$ if we allow the $a_{q}$ to be rational functions (in two variables).

The left hand side of (4) is called a differential polynomial of rank $r$ in $f$. The functions $a_{q}$ are called the coefficients of the differential polynomial. (Later, we will also use differential polynomials with non-rational coefficients.) We also say that the differential equation (4) has rank $r$.

For the proof of our results, we need the following consequences of the chain rule, which are easily proved by induction. Suppose that $h=f(g)$. Then

$$
h^{(n)}=\sum_{w(p)=n} Q_{n, p}[f](g) M_{p}[g],
$$

where $Q_{n, p}$ is a differential polynomial of degree one with constant coefficients. In particular, we have $Q_{n,(n)}[f]=f^{(n)}, Q_{n,(n-2,1)}[f]=(n(n-1) / 2) f^{(n-1)}$, $Q_{n,(1,0, \ldots, 0,1)}[f]=n f^{\prime \prime}, Q_{n,(0, \ldots, 0,1)}[f]=f^{\prime} .\left(\right.$ Here $(1,0, \ldots, 0,1) \in \mathbb{N}_{0}^{n-1}$ and $(0, \ldots, 0,1) \in \mathbb{N}_{0}^{n}$ because $w(p)=n$. $)$ In other words, we have

$h^{(n)}=f^{(n)}(g)\left(g^{\prime}\right)^{n}+\frac{n(n-1)}{2} f^{(n-1)}(g)\left(g^{\prime}\right)^{n-2} g^{\prime \prime}+\cdots+n f^{\prime \prime}(g) g^{\prime} g^{(n-1)}+f^{\prime}(g) g^{(n)}$.

It follows that

$$
M_{q}[h]=\sum_{p \leq q, w(p)=w(q)} N_{q, p}[f](g) M_{p}[g],
$$

where the $N_{q, p}$ are differential polynomials with constant coefficients and the property that every term occuring is of degree $d(q)$. The $N_{q, p}$ can be computed from the $Q_{n, p}$ in an obvious way.

5. Proof of Theorem 1 and 2: Equations for $f$ and $\varphi$. We suppose that $f$ is a transcendental entire function whose iterates are uniformly differentially algebraic and seek a contradiction. The following arguments do, however, also hold for rational $f$, but in this case they represent nothing else than a (suitably modified and rewritten) part of Ritt's proof.

We may assume that $f$ has a repelling fixed point $\zeta$ because otherwise we can replace $f$ by a suitable iterate. We may also assume that $\zeta$ has preimages under $f$ which are different from $\zeta$. There is no loss of generality in assuming that $\zeta=0$ so that $f(0)=0$ and $|\lambda|>1$, where $\lambda=f^{\prime}(0)$, and $f\left(z_{0}\right)=0$ for some 
$z_{0} \neq 0$. Вy $\varphi$ we denote the Poincaré function of $f$ at 0 ; that is, the solution of (2) satisfying $\varphi(0)=0$ and $\varphi^{\prime}(0)=1$. Then

$$
\varphi(z)=\lim _{k \rightarrow \infty} f^{k}\left(\frac{z}{\lambda^{k}}\right)
$$

compare [16, p. 676].

Let now

$$
\sum_{q \leq s} a_{q}(z, u(z)) M_{q}[u](z)=0
$$

be an equation of minimal rank $s$ satisfied by all $f^{k}$. Here the $a_{q}$ are rational in both variables and $a_{s}=1$. We define $g_{k}(z)=f^{k}\left(z / \lambda^{k}\right)$ and note that $\lambda^{k j} g_{k}^{(j)}(z)=\left(f^{k}\right)^{(j)}\left(z / \lambda^{k}\right)$. Thus

$$
\sum_{q \leq s} a_{q}\left(\frac{z}{\lambda^{k}}, g_{k}(z)\right) \lambda^{k w(q)} M_{q}\left[g_{k}\right](z)=0
$$

for all $k$. Now there exist integers $m(q)$ and rational functions $b_{q}(u)$ such that $a_{q}(z, u) \sim z^{m(q)} b_{q}(u)$ as $z \rightarrow 0$. We have $b_{s}=1$ and $m(s)=0$ because $a_{s}=1$. We define $L=\max \{w(q)-m(q)\}$, where the maximum is taken over all $q$ satisfying $a_{q} \neq 0$. Then (7) may be written in the form

$$
\lambda^{k L}\left(\sum_{q \in I} z^{m(q)} b_{q}\left(g_{k}(z)\right) M_{q}\left[g_{k}\right](z)+O\left(\lambda^{-k}\right)\right)=0
$$

where $I$ is the set of indices $q$ such that $L=w(q)-m(q)$. Dividing by $\lambda^{k L}$, taking the limit as $k \rightarrow \infty$, and using (6), we obtain

$$
\sum_{q \in I} z^{m(q)} b_{q}(\varphi(z)) M_{q}[\varphi](z)=0 .
$$

Let now

$$
\sum_{q \leq r} z^{m(q)} c_{q}(\varphi(z)) M_{q}[\varphi](z)=0
$$

be an algebraic differential equation of minimal rank $r$ for $\varphi$ with rational coefficients $c_{q}$ which is normalized by $c_{r}=1$ and $m(r)=0$ and which satisfies the additional condition that $w(q)-m(q)=w(r)$ if $c_{q} \neq 0$. Then $r \leq \max I \leq s$. We define $d=d(r)$ and $w=w(r)$. In view of the results of Section 3, we may assume that the order of the differential equation (8) is at least two. Thus $d<w$.

It follows from (2) that

$$
M_{q}[\varphi]\left(\lambda^{k} z\right)=\lambda^{-k w(q)} M_{q}\left[f^{k}(\varphi)\right](z)
$$

for all $k$ and $q$. We now replace $z$ by $\lambda^{k} z$ in (8), use (2) and (9), multiply the result by $\lambda^{k w}$, and with the abbreviation $F=f^{k}$, we obtain

$$
\sum_{q \leq r} z^{m(q)} c_{q}(F(\varphi(z))) M_{q}[F(\varphi)](z)=0
$$


for all $k$.

We now use (5) and find that the left hand side of (10) is equal to

$$
\begin{aligned}
& \sum_{q \leq r} z^{m(q)} c_{q}(F(\varphi(z))) \sum_{p \leq q, w(p)=w(q)} N_{q, p}[F](\varphi(z)) M_{p}[\varphi](z) \\
= & \sum_{p \leq r} z^{m(p)}\left(\sum_{p \leq q \leq r, w(q)=w(p)} c_{q}(F(\varphi(z))) N_{q, p}[F](\varphi(z))\right) M_{p}[\varphi](z) .
\end{aligned}
$$

Here we have used that $m(p)=m(q)$ if $w(p)=w(q)$. Thus,

$$
\sum_{p \leq r} z^{m(p)} L_{p}[F](\varphi(z)) M_{p}[\varphi](z)=0
$$

with

$$
L_{p}[u]=\sum_{p \leq q \leq r, w(q)=w(p)} c_{q}(u) N_{q, p}[u] .
$$

It is easy to see that $L_{r}[u]=c_{r}(u) N_{r, r}[u]=N_{r, r}[u]=\left(u^{\prime}\right)^{d}$. We define $D_{p}[u]$ by

$$
D_{p}[u](z)=L_{p}[u](z)-c_{p}(z) u^{\prime}(z)^{d} .
$$

Then $D_{r}=0$, and subtracting (8) multiplied by $F^{\prime}(\varphi(z))^{d}$ from (11) we obtain

$$
\sum_{p<r} z^{m(p)} D_{p}[F](\varphi(z)) M_{p}[\varphi](z)=0 .
$$

We proceed to prove that $D_{p}[F]=0$ for all $p$, and thus suppose that this is not the case. We define $h_{p}(z)=z^{m(p)} M_{p}[\varphi](z)$ and $F_{p}=D_{p}[F]$. Then $\sum_{p<r} F_{p}(\varphi) h_{p}=$ 0 and standard results from Nevanlinna theory yield $T\left(r, h_{p}\right)=O(T(r, \varphi))$ as $r \rightarrow \infty$, possibly outside some set of finite measure. Thus Lemma 1 implies that there exist polynomials $P_{p}$ that do not all vanish such that

$$
\sum_{p<r} z^{m(p)} P_{p}(\varphi(z)) M_{p}[\varphi](z)=0 .
$$

This is an algebraic differential equation of rank less than $r$ for $\varphi$, still satisfying $w(p)-m(p)=w$ if $P_{p} \neq 0$ and thus contradicting our choice of $r$. Thus $D_{p}[F]=0$ for all $p$.

We note here that if $f$ and hence $F$ is rational, then we do not need Lemma 1 in order to conclude that $D_{p}[F]=0$ for all $p$.

If $p \neq(w)$, then $D_{p}$ has rank less than $r$. On the other hand, our choice of $s$ implies that the rank of $D_{p}$ is at least $s$, unless $D_{p}=0$. Because $r \leq s$, we conclude that $D_{p}=0$; that is, $D_{p}[u]=0$ for all $u$ (and not only for iterates of $f)$, provided that $p \neq(w)$.

In particular, it follows that $D_{(t)}=0$ if $t \in \mathbb{N}$ and $t \neq w$. Now

$$
D_{(t)}[u](z)=\sum_{q \leq r, w(q)=t} c_{q}(u(z)) M_{q}[u](z)-c_{(t)}(z) u^{\prime}(z)^{d},
$$


and this implies that $w(q)=w$ and $m(q)=0$ if $c_{q} \neq 0$, except possibly if $q=(d)$. Moreover,

$$
D_{(d)}[u](z)=\left(c_{(d)}(u(z))-c_{(d)}(z)\right) u^{\prime}(z)^{d}
$$

so that $c_{(d)}(z)$ is constant, say $c_{(d)}(z)=-a$. We may thus write (8) in the form

$$
\sum_{q \leq r, w(q)=w} c_{q}(\varphi(z)) M_{q}[\varphi](z)=a z^{d-w} M_{(d)}[\varphi](z)
$$

We now show that $d(q) \geq d$ if $c_{q} \neq 0$. Suppose that this is not the case and let $q^{*}$ be the largest rank such that $c_{q^{*}} \neq 0$ and $d^{*}=d\left(q^{*}\right)<d$. Because $N_{q^{*}, q^{*}}[u]=M_{\left(d^{*}\right)}[u]=\left(u^{\prime}\right)^{d^{*}}$, we have

$$
L_{q^{*}}[u]=c_{q^{*}}(u)\left(u^{\prime}\right)^{d^{*}}+\sum_{q^{*}<q \leq r, w(q)=w(p)} c_{q}(u) N_{q, q^{*}}[u] .
$$

We observe that if $q>q^{*}$, then all monomials in $N_{q, q^{*}}[u]$ contain higher derivatives of $u$ so that no term in the sum on the right hand side can cancel the term $c_{q^{*}}(u)\left(u^{\prime}\right)^{d^{*}}$. Because $d^{*} \neq d$ we obtain a contradiction to the equation $D_{q^{*}}=0$. Thus, $d(q) \geq d$ for all $q$.

6. The reduction of the rank of the equation. We now use the equations $D_{p}=0$ to determine the possible forms of (12).

First we show that $r=\left(r_{1}, r_{2}, \ldots, r_{n}\right)$ has the form $r=\left(r_{1}, 0, \ldots, 0,1\right)$. Suppose that this is not the case and define $p^{\prime}=(w-n, 0, \ldots, 0,1) \in \mathbb{N}_{0}^{n}$. We observe that $N_{r, p^{\prime}}$ contributes a term of rank $\left(r_{1}+n, r_{2}, \ldots, r_{n-1}, r_{n}-1\right)$ to $D_{p^{\prime}}$, while all other terms $N_{q, p^{\prime}}$ contribute only terms of smaller rank to $D_{p^{\prime}}$. This contradicts the equation $D_{p^{\prime}}=0$. Thus $r=\left(r_{1}, 0, \ldots, 0,1\right)$.

Now we show that $n \leq 3$ so that $r=\left(r_{1}, 1\right)$ or $r=\left(r_{1}, 0,1\right)$. In order to do this we suppose that $n>3$ and define $p^{\prime \prime}=\left(r_{1}+1,0, \ldots, 0,1\right) \in \mathbb{N}_{0}^{n-1}$ and $q^{\prime}=\left(r_{1}-1,1,0, \ldots, 0,1\right) \in \mathbb{N}_{0}^{n-1}$. We observe that $N_{r, p^{\prime \prime}}$ contributes $n M_{\left(r_{1}, 1\right)}$ to $D_{p^{\prime \prime}}$ while $c_{q^{\prime}} N_{q^{\prime}, p^{\prime \prime}}$ contributes $c_{q^{\prime}} M_{\left(r_{1}, 1\right)}$ to $D_{p^{\prime \prime}}$. All other $N_{q, p^{\prime \prime}}$ with $w(q)=w$ and $d(q) \geq d$ do not contain the monomial $M_{\left(r_{1}, 1\right)}$. Thus $D_{p^{\prime \prime}}=0$ implies that $c_{q^{\prime}}+n=0$.

Next we define $q^{\prime \prime}=\left(r_{1}, 0, \ldots, 0,1\right) \in \mathbb{N}_{0}^{n-1}$ and note that $N_{r,\left(r_{1}+n-2,1\right)}$ contributes $(n(n-1) / 2) M_{q^{\prime \prime}}$ to $D_{\left(r_{1}+n-2,1\right)}$ while $N_{q^{\prime},\left(r_{1}+n-2,1\right)}$ contributes $c_{q^{\prime}} M_{q^{\prime \prime}}$ to $D_{\left(r_{1}+n-2,1\right)}$. All other contributions are of smaller rank and we find that $c_{q^{\prime}}+n(n-1) / 2=0$. Altogether we thus have $n=n(n-1) / 2$. This contradicts the assumption $n>3$. Thus $n \leq 3$.

Suppose first that $n=2$ so that $r=\left(r_{1}, 1\right)$. If $q<r$ and $c_{q} \neq 0$, then $q=\left(q_{1}\right)$ where $q_{1}=d=r_{1}+1$ or $q_{1}=w(q)=r_{1}+2$. Thus (12) has the form

$$
M_{r}[\varphi](z)+c_{\left(r_{1}+2\right)}(\varphi(z)) M_{\left(q_{1}+2\right)}[\varphi](z)=\frac{a}{z} M_{\left(q_{1}+1\right)}[\varphi](z) .
$$


We divide this equation by $\varphi^{\prime}(z)^{r_{1}}$ and using the abbreviation $A=-c_{\left(r_{1}+2\right)}$ we obtain

$$
\varphi^{\prime \prime}(z)=A(\varphi(z)) \varphi^{\prime}(z)^{2}+\frac{a}{z} \varphi^{\prime}(z) .
$$

Suppose next that $n=3$ so that $r=\left(r_{1}, 0,1\right)$. If $q<r$ and $c_{q} \neq 0$, then $q=(d)=\left(r_{1}+1\right)$ or $w(q)=w=r_{1}+3$ and $d(q) \geq d=r_{1}+1$. This shows that $c_{q}=0$ except possibly if $q=\left(r_{1}-1,2\right), q=\left(r_{1}+1,1\right), q=\left(r_{1}+3\right)$, or $q=\left(r_{1}+1\right)$.

We observe that $N_{r,\left(r_{1}+1,1\right)}=3 M_{\left(r_{1}, 1\right)}, N_{\left(r_{1}-1,2\right),\left(r_{1}+1,1\right)}=2 M_{\left(r_{1}, 1\right)}$, and $N_{\left(r_{1}+1,1\right),\left(r_{1}+1,1\right)}=M_{\left(r_{1}+2\right)}$. Thus the equation $D_{\left(r_{1}+1,1\right)}=0$ takes the form

$$
\left(3+2 c_{\left(r_{1}-1,2\right)}\right) M_{\left(r_{1}, 1\right)}+c_{\left(r_{1}+1,1\right)} M_{\left(r_{1}+2\right)}-c_{\left(r_{1}+1,1\right)} M_{\left(r_{1}+1\right)}=0 .
$$

We conclude that $c_{\left(r_{1}-1,2\right)}=-\frac{3}{2}$ and $c_{\left(r_{1}+1,1\right)}=0$. Using this in (12), dividing by $\varphi^{\prime}(z)^{r_{1}+1}$, and defining $A=-c_{\left(r_{1}+3\right)}$ we find that

$$
S \varphi(z)=A(\varphi(z)) \varphi^{\prime}(z)^{2}+\frac{a}{z^{2}},
$$

where

$$
S \varphi=\frac{\varphi^{\prime \prime \prime}}{\varphi^{\prime}}-\frac{3}{2}\left(\frac{\varphi^{\prime \prime}}{\varphi^{\prime}}\right)^{2}=\left(\frac{\varphi^{\prime \prime}}{\varphi^{\prime}}\right)^{\prime}-\frac{1}{2}\left(\frac{\varphi^{\prime \prime}}{\varphi^{\prime}}\right)^{2}
$$

denotes the Schwarzian derivative of $\varphi$.

7. Completion of the proof. We are left with the differential equations (13) and (14). As noted by Ritt, it can be shown by elementary differentiation that any solution of (13) also satisfies an equation of the form (14). Thus it suffices to consider (14). Ritt, using the existence of $z_{0} \neq 0$ satisfying $f\left(z_{0}\right)=0$, showed that there exists a positive integer $p$ such that $\psi(z)=\varphi\left(z^{p}\right)$ satisfies $S \psi=A(\psi)\left(\psi^{\prime}\right)^{2}$ and that this implies that any branch of the inverse function of $\psi$ is a linear function of any other branch. As he notes, this allows the explicit determination of all possible functions $\psi$ and $\varphi$ in terms of exponential, trigonometric, and elliptic functions. Here we only have to note that the above property of the branches of the inverse function clearly implies that $\psi$ and hence $\varphi$ have finite order, which is impossible by Lemma 2 . .

\section{Remarks.}

(1) We cannot hope to further reduce the rank of the equation (14) using the methods of Section 6. In fact, a simple computation shows that if $f$ satisfies

$$
S u(z)=A(u(z)) u^{\prime}(z)^{2}-A(z),
$$


then so does any iterate of $f$. And the solutions of $S \psi=A(\psi)\left(\psi^{\prime}\right)^{2}$ conjugate the solutions of (15) to linear transformations. The reasoning above shows, however, that (15) does not have transcendental entire solutions. We remark that the monomials satisfy (15) with $A(u)=-1 /\left(2 u^{2}\right)$ while the Chebychev polynomials satisfy (15) with $A(u)=-\left(u^{2}+2\right) /\left(2\left(u^{2}-1\right)^{2}\right)$.

(2) As already mentioned, the arguments of Section 5 and Section 6 remain valid for rational $f$. They show that differentially algebraic Poincaré functions to rational functions satisfy a first order algebraic differential equation or a differential equation of the form (13) or (14). Ritt showed how this permits to determine them explicitly.

\section{REFERENCES}

[1] I. N. Baker, Repulsive fixpoints of entire functions. Math. Z. 104 (1968), $252-256$

[2] A. F. Beardon, Iteration of rational functions. New York Berlin Heidelberg: Springer 1991

[3] P.-G. Becker and W. Bergweiler, Hypertranscendency of local conjugacies in complex dynamics. Math. Ann. 301 (1995), 463-468.

[4] W. Bergweiler, Periodic points of entire functions: proof of a conjecture of Baker. Complex Variables Theory Appl. 17 (1991), 57-72

[5] M. Boshernitzan and L. A. Rubel, Coherent families of polynomials. Analysis 6 (1986), 339-389

[6] W. D. Brownawell, On the factorization of partial differential equations. Can. J. Math. 39 (1987), 825-834

[7] L. Carleson and Th. W. Gamelin, Complex dynamics. New York Berlin Heidelberg: Springer 1993

[8] J. Clunie, The composition of entire and meromorphic functions. In: $\mathrm{H}$. Shankar (ed.), Mathematical essays dedicated to A. J. Macintyre, pp. 7592. Athens: Ohio University Press 1970

[9] F. Gross and C. F. Osgood, A simpler proof of a theorem of Steinmetz. J. Math. Anal. Appl. 143 (1989), 490-496

[10] F. Gross and C. F. Osgood, An extension of a theorem of Steinmetz. J. Math. Anal. Appl. 156 (1991), 290-294

[11] F. Gross and C. F. Osgood, Finding all solutions related to Steinmetz's theorem. J. Math. Anal. Appl. 164 (1992), 417-421

[12] W. K. Hayman, Meromorphic functions. Oxford: Clarendon Press 1964

[13] G. Jank and L. Volkmann, Einführung in die Theorie der ganzen und meromorphen Funktionen mit Anwendungen auf Differentialgleichungen. Basel Boston Stuttgart: Birkhäuser 1985

[14] G. Kønigs, Recherches sur les integrales de certains équations fonctionelles. Ann. Sci. École Norm. Sup. (3) 1 (1884), 1-41

[15] H. Poincaré, Sur une classe nouvelle des transcendantes uniformes. J. Math. Pures Appl. (4) 6 (1890), 313-365

[16] J. F. RitT, Transcendental transcendency of certain functions of Poincaré. Math. Ann. 95 (1925/26), 671-682

[17] L. A. Rubel, Some research problems about algebraic differential equations. Trans. Amer. Math. Soc. 280 (1983), 43-52

[18] L. A. Rubel, A survey of transcendentally transcendental functions. Amer. Math. Monthly 96 (1989), 777-788 
[19] L. A. Rubel, Some research problems about algebraic differential equations II. Illinois J. Math. 36 (1992), 659-680

[20] E. Schröder, Über iterirte Functionen. Math. Ann. 3 (1871), 296-322

[21] N. Steinmetz, Über faktorisierbare Lösungen gewöhnlicher Differentialgleichungen. Math. Z. 170 (1980), 169-180

[22] N. Steinmetz, Rational iteration. Berlin: Walter de Gruyter 1993

23] G. VAliron, Sur les fonctions entières d'ordre nul et d'ordre fini et en particulier les fonctions a correspondance régulière. Ann. Fac. Sci. Toulouse (3) 5 (1913), 117-257

[24] G. Valinon, Lectures on the general theory of integral functions. Toulouse: Édouard Privat 1923

Lehrstuhl II für Mathematik

RWTH Aachen

D-52056 Aachen, Germany

Present AdDress: Fachbereich Mathematik

Technische Universität Berlin

Sekr. MA 8-2

Straße des 17. Juni 136

D-10623 Berlin, Germany

E-mail: bergweil@math.tu-berlin.de

Received: September 26th, 1994. 